\title{
A Novel Modular Multilevel Converter Based on Interleaved Half-Bridge Submodules
}

This paper was downloaded from TechRxiv (https://www.techrxiv.org).

LICENSE

CC BY-NC-SA 4.0

SUBMISSION DATE / POSTED DATE

$20-07-2021 / 26-07-2021$

\section{CITATION}

Viatkin, Aleksandr; Ricco, Mattia; Mandrioli, Riccardo; Kerekes, Tamas; Teodorescu, Remus; Grandi, Gabriele (2021): A Novel Modular Multilevel Converter Based on Interleaved Half-Bridge Submodules. TechRxiv. Preprint. https://doi.org/10.36227/techrxiv.15022920.v1

$\mathrm{DOI}$

10.36227/techrxiv.15022920.v1 
(C) 2021 IEEE. Personal use of this material is permitted. Permission from IEEE must be obtained for all other uses, in any current or future media, including reprinting/republishing this material for advertising or promotional purposes, creating new collective works, for resale or redistribution to servers or lists, or reuse of any copyrighted component of this work in other works.

\section{A Novel Modular Multilevel Converter Based on Interleaved Half-Bridge Submodules}

Viatkin A.; Ricco M.; Mandrioli R.; Kerekes T.; Teodorescu R.; Grandi G.

last update: 20 July, 2021

\section{Post conference paper:}

A. Viatkin, M. Ricco, R. Mandrioli, T. Kerekes, R. Teodorescu and G. Grandi, "Modular Multilevel Converters Based on Interleaved Half-Bridge Submodules," 2021 22nd IEEE International Conference on Industrial Technology (ICIT), 2021, pp. 440-445, doi: 10.1109/ICIT46573.2021.9453643.

Aleksandr Viatkin, Mattia Ricco, Riccardo Mandrioli, and Gabriele Grandi are with the Department of Electrical, Electronic, and Information Engineering, University of Bologna, Bologna, Italy (e-mail: aleksandr.viatkin2@ unibo.it; mattia.ricco@unibo.it; riccardo.mandrioli4@unibo.it; gabriele.grandi@unibo.it). Tamás Kerekes and Remus Teodorescu are with the Department of Energy Technology, Aalborg University, Aalborg, Denmark (e-mail: tak@et.aau.dk; ret@et.aau.dk). 


\title{
A Novel Modular Multilevel Converter Based on Interleaved Half-Bridge Submodules
}

\author{
Aleksandr Viatkin, Student Member, IEEE, Mattia Ricco, Senior Member, IEEE, \\ Riccardo Mandrioli, Student Member, IEEE, Tamás Kerekes, Senior Member, IEEE, \\ Remus Teodorescu, Fellow, IEEE, and Gabriele Grandi, Senior Member, IEEE
}

\begin{abstract}
Post Conference Paper: A. Viatkin, M. Ricco, R. Mandrioli, T. Kerekes, R. Teodorescu and G. Grandi, "Modular Multilevel Converters Based on Interleaved Half-Bridge Submodules," 2021 22nd IEEE International Conference on Industrial Technology (ICIT), 2021, pp. 440-445, doi: 10.1109/ICIT46573.2021.9453643.
\end{abstract}

\begin{abstract}
A new Modular Multilevel Converter with Interleaved half-bridge Sub-Modules (ISM-MMC) is proposed in this paper. The ISM-MMC exhibits a higher modularity and scalability in terms of current ratings with respect to a conventional MMC, while preserves the typical voltage level adaptiveness. The ISM-MMC brings the known advantages of classical MMC to low-voltage, high-current applications making it a novel candidate for the sector of ultra-fast chargers for all types of electrical vehicles (EV). This advanced topology makes it possible to easily reach charging power of the EV charging system up to $4.5 \mathrm{MW}$ and beyond with low-voltage supply. To operate the new converter, a hybrid modulation scheme that helps to exploit advantages of the interleaving scheme, is implemented, and explained in this paper. It has been verified that the typical MMC control methods are still applicable for ISMMMC. A comparative study between classical MMC and ISM-MMC configurations in terms of output characteristics and efficiency is also given. Furthermore, it has been demonstrated that the number of ac voltage levels is synthetically multiplied by the number of interleaved halfbridge legs in submodules. Numerical simulations and Hardware-in-the-Loop tests are carried out to demonstrate the feasibility of the proposed topology and implemented modulation scheme.
\end{abstract}

Index Terms - modular multilevel converters (MMC), interleaved, pulse width modulation, charging stations, electric vehicles.

\section{INTRODUCTION}

$\mathrm{M}$ ODULAR multilevel converters (MMCs) have been winning over conventional solutions in many high- and medium-voltage applications owing to their capability in transformerless ac-dc and dc-ac conversion, while introducing lower harmonic pollution and having higher efficiency. While MMC has become the worldwide standard for high-voltage dc transmission [1], it has also been investigated in the power electronic transformer applications [2], medium-voltage motor drives, frequency change systems [3], etc. Depending on the application, the structure of submodules (SMs) is mainly halfbridge (HB) or full-bridge (FB), while many other types of SMs have been reported in the literature [3]. While voltage ratings of MMCs can vary in a great extent, current ratings instead are normally limited by the maximum current that components (mainly power switches) can handle, taking into account safety margins. In this context, the power capability of the classical MMC is limited once the voltage level was fixed. Some possibilities to boost current capacity of the classical MMC have been already discussed in [4], [5], exploiting current partitioning through parallel connection of converters' legs, arms (branches) or power modules in each MMC cell. However, the presented solutions do not exploit the interleaving scheme and hardly scalable when converter is already built/structured since it involves modifications on the converter level.

The interleaved concept has been thoroughly studied in the context of two-level dc-ac and dc-dc converters [6], providing opportunity not only to share the total current among interleaved units but also enhancing quality of input/output waveforms. For instance, authors in [6] demonstrated the possibility to obtain a ripple-free output current in interleaved dc-dc converters. On the other hand, this approach has been limited in MMC applications. In [7] authors proposed a dc-dc interleaved MMC for PV applications. Although, the proposed topology is named as "modular multilevel converter" it only partially resembles to a classical MMC structure, sharing floating capacitors between adjacent submodules. By bringing together the best practice of interleaving theory and current partitioning in a classical MMC structure, the ISM-MMC was initially introduced in [8].

There are several trending low-voltage, high-power applications that can benefit from ISM-MMC architecture, namely converters in ac or dc traction power supplies and ultrafast electric vehicle (EV) charging infrastructure. However, this list is not limited by low-voltage level. In fact, since the new converter topology is easily scalable in both voltage and current ratings, it can be used in a wide range of voltage and current levels. For instance, at this moment several charging systems are available on the market (e.g., GB/T, New GB/T, CHAdeMO, CCS1, CSS2, Tesla) having maximum charging power in between 237.5 - $900 \mathrm{~kW}$ range. Furthermore, a new High Power Commercial Vehicle Charging (HPCVC) standard is currently under development, which will level up the power delivery up to 4.5 MW. For this paper, a well-adopted commercial infrastructure for ultrafast EV charging with output power 180 $\mathrm{kW}$ has been selected as a reference application. 


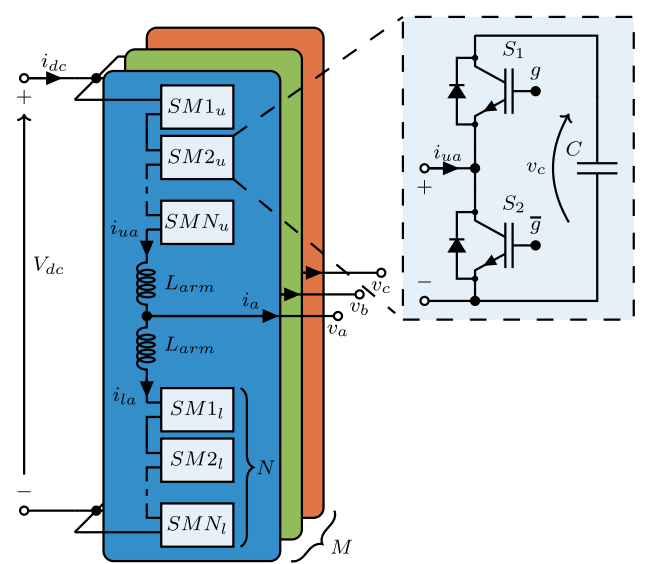

(a)

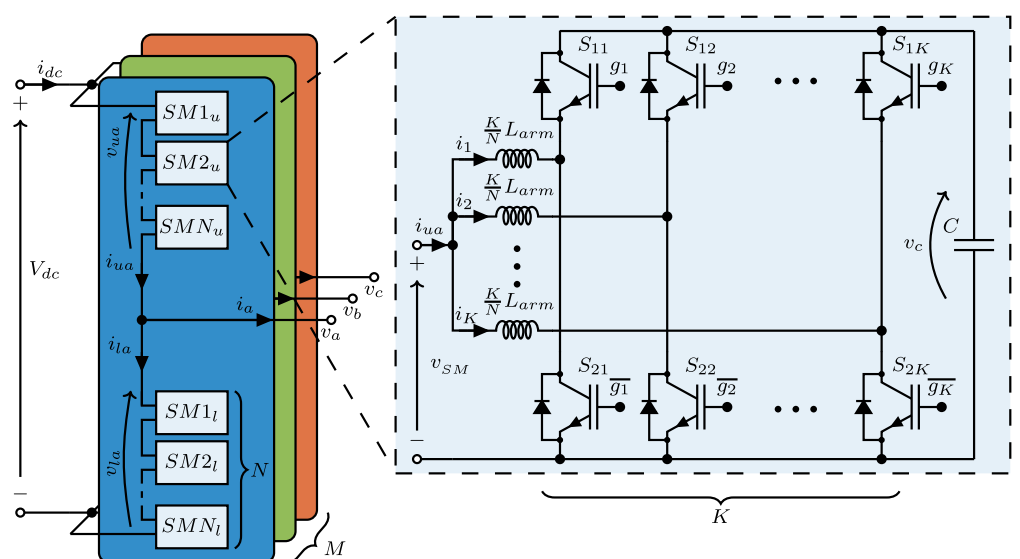

(b)

Fig. 1. Scheme of (a) the standard MMC converter with half-bridge submodule and (b) the proposed ISM-MMC converter with interleaved half-bridge submodule.

To the best of the authors' knowledge, all the previous publications regarding the MMC structure have focused on interleaving either at the leg or arm levels, and none, apart from [8], were considering the interleaving concept at the submodule level. The main contribution of the current work concerns some missing aspects and developments of [8], namely, performance comparison between the classical MMC and ISM-MMC structures, as well as an implementation of the closed-loop strategy for ISM-MMC and analysis of its dynamic behavior.

The paper is structured as follows. Section II introduces the ISM-MMC topology, providing essential mathematical models. The closed-loop control methods and modulation schemes are discussed in Section III. Verification of ISM-MMC concept is supported by numerical simulation and hardware-in-the-loop (HIL) in Sections IV and V, respectively. The comparison of ISM-MMC with the classical MMC configurations is given in Sections VI. Finally, conclusions are drawn in Section VII.

\section{Proposed Topology}

A structural representation of an $M$ phase (typically 3 phase) MMC using a half-bridge (HB) submodule configuration is shown in Fig. 1a. This MMC employs two arm arrangement in each phase leg, commonly labeled as the upper $(u)$ and lower $(l)$ arms. Each MMC arm consists of $N$ series connected power submodules and an arm inductor. Every SM is made up by a halfbridge leg joined in parallel with a capacitor. The output terminals of the submodule are the midpoint of the half-bridge leg and one of the sides of the capacitor (here low side is taken by default). The voltage level and power capacity of this type of MMC can be generally increased by the series connection of power submodules in each arm. As it was explained in [8] the arm inductor can be evenly distributed among the arm composing submodules without affecting the equivalent circuit of the whole converter. Having an inductor in each SM, it can be further split into $K$ equally sized parallel inductors. Then, by connecting each one of these inductors to the midpoint of a dedicated half-bridge leg and linking their output and dc terminals the interleaved configuration of a submodule with $K$ legs can be derived. The proposed MMC with interleaved half-bridge submodules, labeled as ISM-MMC, is illustrated in Fig. 1b. It should be noted that the interleaved half-bridge legs on the dc side share a common floating capacitor. This fact does not introduce extra complexity for the capacitor voltage balancing algorithms that are well established for the classical MMC. The current rating of 'inductor - HB-leg' units in the proposed submodule can be $K$ times lower in comparison with switches and arm inductor in a classical MMC (cf. Fig. 1a). Alternatively, the total rated current of the new SM can be $K$ times higher than the classical HB-based $\mathrm{SM}$, preserving properties of individual HB-legs. Therefore, ISM-MMC introduces an additional way for increasing power capacity of the standard MMC structure by stepping up both voltage and current levels. In this context, the proposed ISMMMC is well suited for all voltage levels high-current applications. The concepts of power scalability and/or power partitioning, discussed in [4], [5], with ISM-MMC gain an extra degree of freedom in comparison with classical MMCs, namely expanding on the submodule level. Since the degree of modularity in each of MMC levels: number of phases, number of series connected cells (SMs) and number of parallel (interleaved) HB legs in SMs are independent from each other, these three axes can be considered as orthogonal to one another, enabling a representation as shown in Fig. 2. Here, each cube represents a specific design with a certain degree of modularity in each of the axes.

For instance, an element close to origin would represent a design with low-level of modularity in all axes, i.e., a singlephase, single-cell MMC. On the other hand, an element distant from the origin would represent a highly expansive structure, i.e., a multi-phase, multi-cell ISM-MMC.

The proposed ISM-MMC has an additional feature that has not been discussed so far, namely the possibility not only sharing high-current among the parallel HB legs in each SM but also to further enhance the quality of output voltage waveforms by applying the interleaving concept. This ISM-MMC property leads to a very modest filtering requirements on the grid side.

Assuming a balanced and constant dc-link bus voltage equal to $V_{d c}$, the per phase relation among each output $x^{\text {th }}$ phase (for three-phase system $x=a, b, c)$ voltage, corresponding arm voltages and dc-link voltage in ISM-MMC can be expressed as: 


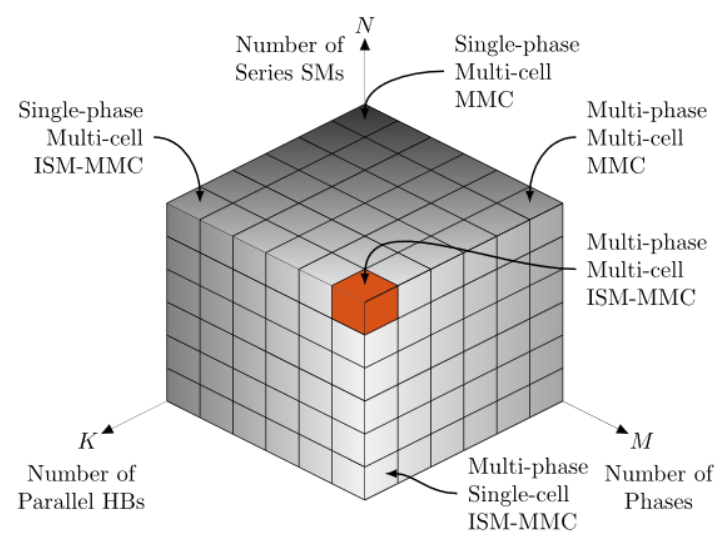

Fig. 2. Graphical representation of the three main degrees of modularity of ISM-MMC topologies.

$$
\frac{V_{d c}}{2}-v_{u}=v, \quad-\frac{V_{d c}}{2}+v_{l}=v
$$

being $v_{u}$ upper and $v_{l}$ lower arm voltages, $v$ the output phase voltage. Here and throughout the whole paper, for simplicity, the phase label $(x)$ is generally omitted unless it is strictly necessary. The presented equations are derived per phase.

The arm voltages $v_{u}$ and $v_{l}$, which are measured between the midpoint of the converter's arm and the corresponding dc rails, can be represented as a sum of composing submodule voltages:

$$
v_{u, l}=\left.\sum_{n=1}^{N} v_{S M n}\right|_{u, l}
$$

where $\left.v_{S M n}\right|_{u, l}$ is the $n^{\text {th }}$ submodule's voltage of the upper or lower arm. It can be derived as:

$$
v_{S M n}=\frac{1}{K}\left[L \sum_{k=1}^{K} \frac{d i_{k, n}}{d t}+R \sum_{k=1}^{K} i_{k, n}+z_{n} v_{c, n}\right]
$$

where $K$ is the number of legs inside the submodule ( $K=1$ in the case of the classical MMC Fig. 1a, $K>1$ in case of the interleaved configuration Fig. 1b) and $n$ represents an ordinal number of the corresponding $n^{\text {th }} \mathrm{SM}$ of the upper or lower arm. Parameters $L$ and $R$ are the inductance and internal resistance of a leg inductor inside each SM, respectively. The term $i_{k, n}$ is the current of the $k^{\text {th }}$ leg inside the $n^{\text {th }} \mathrm{SM}$. It is worth to note that $i_{k, n}$ is equal to $i_{u, l}$ in case of classical MMC being the SM composed of only one HBleg. Finally, $v_{c}$ and $z$ are the capacitor voltage and the corresponding number of HB legs in the SM where the top switch is "on". The latter can be evaluated as the sum of logical gate signals $g_{k, n}$ (having value either 0 or 1 ) in $n^{\text {th }}$ submodule:

$$
\mathrm{z}_{n}=\sum_{k=1}^{K} g_{k, n}
$$

As it can be noted from (3), the aforementioned enhancement effect of converter's ac voltage is achieved due to the fact that the voltage across ac terminals of each submodule is actually multilevel itself. In fact, in total, there are $K+1$ voltage levels ranging between " 0 " and " $v_{c}$ ". Having identical inductor values and in accordance with Millman's theorem the voltage across ac terminals is simply the average of composing pole voltages of the parallel branches. Depending on state of the legs' switches, the corresponding pole voltage can be either " 0 " or " $v_{c}$ ". By applying the interleaving concept, the averaging of pole voltages will result in multilevel voltage structure with the following states 0 , $v_{c} / K, 2 v_{c} / K \ldots(K-1) v_{c} / K, v_{c}$. Depending on the direction of the arm current, the capacitor voltage either increases or decreases. When all top switches are "off," the ac output submodule voltage is equal to " 0 ." In this mode, the capacitor voltage remains constant, irrespective of the current direction.

The output $(i)$ and circulating $\left(i_{c}\right)$ currents in the ISM-MMC are alike in classical MMCs and they can be defined as:

$$
i=i_{u}-i_{l}, \quad i_{c}=\frac{i_{u}+i_{l}}{2}
$$

where $i_{u}$ and $i_{l}$ are the upper and lower arm currents, respectively.

Bearing in mind that the sum of the individual leg currents in (3) is equal to the arm current $i_{u, l}$ and by combining (1), (2), (3) and (5) the two equations that govern the output and circulating currents are:

$$
\begin{gathered}
\frac{N L}{2 K} \frac{d i}{d t}=\frac{1}{2 K} \sum_{n=1}^{N}\left[z_{l, n} v_{c l, n}-z_{u, n} v_{c u, n}\right]-\frac{N R}{2 K} i-v \\
\frac{N L}{K} \frac{d i_{c}}{d t}=\frac{V_{d c}}{2}-\frac{1}{2 K} \sum_{n=1}^{N}\left[z_{l, n} v_{c l, n}+z_{u, n} v_{c u, n}\right]-\frac{N R}{K} i_{c}
\end{gathered}
$$

By matching inductances $L$ and internal resistances $R$ of the individual inductors in SMs to $K / N$ times of the arm inductance $L_{a r m}$, as depicted in Fig. $1 \mathrm{~b}$, the identical equivalent circuit characteristics of the classical MMC topology can be achieved.

$$
L=\frac{K}{N} L_{\text {arm }}, \quad R=\frac{K}{N} R_{\text {arm }}
$$

In this context, substituting (7) into (6) will lead to an identical averaged dynamic model of the proposed ISM-MMC with respect to a classical MMC [9]. In fact, as it will be shown later, only few modifications are required in the control method of ISM-MMC in comparison with standard MMC. This characteristic makes ISM-MMC solution very attractive for retrofitting already built MMCs when higher power capacity of the new converter is required. In addition to that by taking advantage of a proper modulation for driving interleaved HB-legs of each SM, the output characteristics of the converter can be significantly improved.

\section{CONTROL AND MODULATION}

The proposed ISM-MMC has primary and secondary control objectives, in the same manner as the classical MMC. The submodule capacitor voltage balancing, and output current control are the primary objectives that directly relate to the operation of ISM-MMC. At the same time, the circulating current control is a secondary objective and associated with the size, reliability, and the efficiency of a converter. Unlike standard MMC, the ISM-MMC has an additional secondary control objective to deal with, namely equal current sharing among interleaved HB-legs in each SM. This problem becomes quite challenging with an increase of both series connected submodules and interleaved HB-legs. Therefore, this subject will be discussed in great detail in a separate work. In fact, the current balancing of the interleaved legs is a decoupled control task that is associated with performance of a single submodule and with acceptable level of the unbalance it does not affect the operational behavior of the whole converter (the output characteristics remain unchanged). However, it should be noted that the obvious drawback of such unsupervised SM currents operation is a need in oversizing composed SM components. All these aspects will 


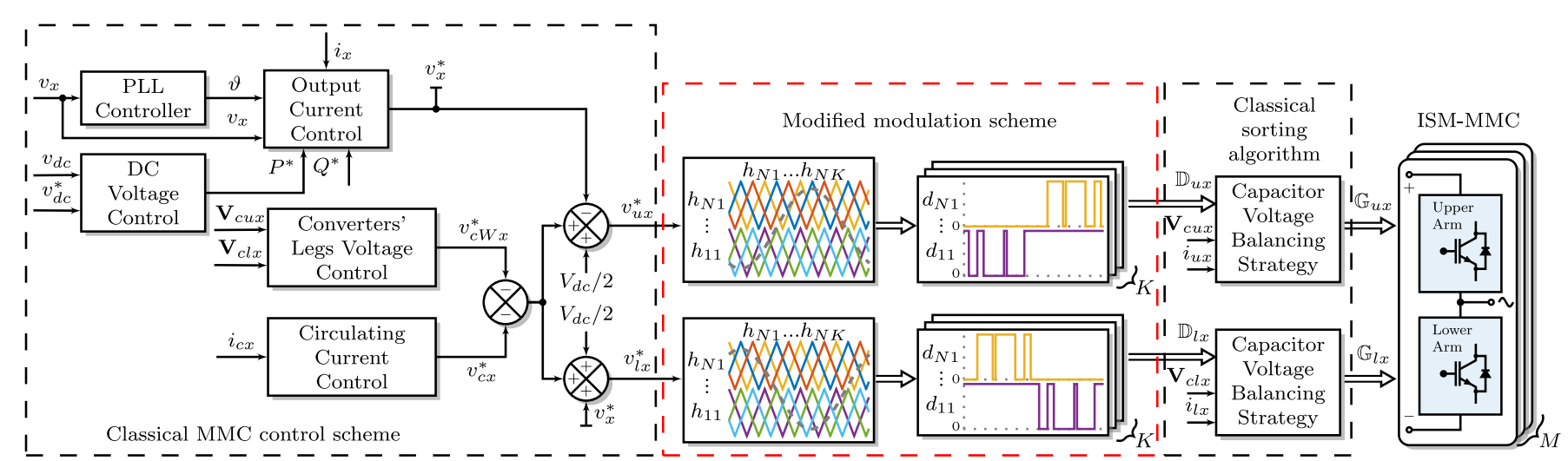

Fig. 3. Block diagram of implemented control method of the proposed ISM-MMC.

be addressed in future works. Instead, this section deals with the implementation and performance of classical MMC control methods that perfectly fit for the proposed ISM-MMC with small modifications that are discussed below.

\section{A. Control method}

Literature discusses the functionality of classical control methods for the MMC topology in great detail. For example, [9], [10] describe the operating and control principle very clearly. Therefore, and for the sake of conciseness, here a brief overview is given only.

The general block diagram of implemented control methods for the proposed ISM-MMC is shown in Fig. 3. This control structure is designed for a front-end converter that interfaces ac grid and can be used in high-power applications, i.e., electric vehicles charging. It includes independent control approaches that are used to regulate dc-link voltage, the submodule capacitors voltage, output currents, and circulating currents. A simple proportional integral (PI) controller is adopted to regulate the dc-link voltage as the outer loop, and it provides the active power reference to the output current regulator that works as the inner loop. In addition to the active power, the reactive power reference can be added to control level or reactive power in the grid. The output and circulating currents are adjusted using closed-loop controllers (cf. Fig. 3), which generate control commands $v_{x}^{*}$ (reference of the output voltage) and $v_{c x}^{*}$ (compensating reference of the circulating current regulator), respectively. Both controllers are based on proportional resonant (PR) control strategy, capable to effectively track sinusoidal reference and reject disturbance with a low computation burden [9], [10]. The submodule capacitors voltage control aims to maintain the capacitor's voltage at an identical value within the arm. The voltage balancing can be attained either at control or modulation stage. At the control stage an additional closed-loop controller is required while implementing this regulation at modulation stage involves balancing logical functions [11], [12]. In the classical balancing method, associated with level-shifted PWM, the capacitor voltages (vectors $\mathbf{V}_{c u x}, \mathbf{V}_{c l x}$ ) within an arm are sorted either in ascending or descending order in accordance with the direction of the arm current. Then, the input gate signals are rearranged in agreement with the sorted capacitor voltages and the direction of the arm current. This algorithm operates directly on the generated set of PWM signals $\left(2 \mathrm{D}\right.$ arrays $\mathbb{D}_{u x}$, $\mathbb{D}_{l x}$ ), which are result of comparison between modulating signals and carriers. The output of this block is a set of logical gate signals ( $2 \mathrm{D}$ arrays $\mathbb{G}_{u x}, \mathbb{G}_{l x}$ ) that drive switches. The algorithm itself does not require a modification to meet the balancing requirements for an ISM-MMC since interleaving of HB-legs inside a submodule does not change its equivalent circuit. Nevertheless, the number of commutating switches is increased, and interleaving angles are applied. Likely, the interleaving concept is working entirely within a submodule, therefore, depending on the sorting algorithm action (bypass or insert the submodule), the group of gate signals can be swapped with similar group from another submodule that should be either inserted or bypassed. In this way the voltage balancing algorithm is irrespective of the number of interleaved HB-legs inside the submodule. Another type of control focuses to maintain the sum of capacitor voltages within each converters' arm, at their desired common value, $V_{d c}$. This closed-loop controller generates a compensating signal $v^{*}{ }_{C W x}$, which is added to the upper and lower arm modulation signals. This compensating signal is composed by action of the total $\left(W_{\Sigma}\right)$ and imbalance $\left(W_{\Delta}\right)$ arm energies regulators [9].

\section{B. Modulation}

Many modulation schemes have been adopted to MMC based topologies. Among them the most widely employed modulation techniques can be categorized as multilevel carrier-based PWM techniques with either level-shifted (LS-PWM) or phase-shifted (PS-PWM) carriers [13], [14], staircase waveform modulations [15], [16], and space vector modulation (SVM) [17]-[19].

Staircase modulation methods feature fundamental switching frequency, reduced switching losses and simple realization, however it comes with the price of increased harmonic distortion of the output voltage and current waveforms. The quality of output waveforms can be improved by increasing the number of submodules, which is the case for an MMC-based high voltage applications. These staircase methods mainly include selective harmonic elimination (SHE) scheme and nearest level modulation (NLM). The NLM approach is computationally less complex, however its performance is significantly affected by the sorting algorithm and sampling frequency, especially when the number of submodules is low [16]. The SHE scheme requires off-line computation of a large number of switching angles, which increase the computational complexity with the growth of the number of voltage levels.

The SVM directly controls the line-to-line voltages of a modular multilevel converter and allows generating the phase voltages implicitly. In this way SVM eliminates the influence of commonmode voltages and provides more flexibility (i.e., redundant switching sequences) to optimize switching pattern [18]. 
Nevertheless, the SVM method is difficult to implement for a converter from the MMC family with many voltage levels due to high computational burden.

On the other hand, carrier-based modulation schemes are widely applied to control multilevel power converters due to their simple implementation and ease of extension to higher number of voltage levels. In PS-PWM, the triangular carriers with an identical magnitude are horizontally biased, while in LS-PWM they are disposed vertically. The LS-PWM can be further classified based on the phase relationship between the adjacent carriers into phase disposition (PD), phase opposition disposition (POD), alternate phase opposition disposition (APOD) and other hybrid schemes. The carrier-based modulations usually fall into the high switching frequency category, therefore, have higher switching losses in comparison with staircase modulation schemes. Moreover, an accurate synchronization between the carriers is essential to generate high-quality voltage and current waveforms [14].

Similarly, the interleaving modulation methods have been wellreported in literature as well. Most of the attention in this regard has been drawn to a phase-shift in the operation of the parallel branches, generally achieved through PS-PWM [20] or SVM [21]. Another trending modulation strategy to handle interleaving in VSCs is the LS-PWM [22], [23].

To drive the proposed ISM-MMC, a hybrid modulation scheme is implemented. It is composed of LS-PWM for synthesizing voltage levels given by series connected SMs whether PS-PWM scheme handles interleaving of parallel HB-legs within each SM. A classical MMC with $N$ SMs per arm (cf. Fig. 1a) can provide either $N+1$ or $2 N+1$ levels in the output voltage, depending on whether transitions in the upper and lower arms are synchronized or not (arm interleaved scheme). In relation to LS-PWM N+1 levels correspond to APOD scheme, while $2 N+1$ levels can be generated with PD approach. In the current work PD LS-PWM has been selected to maximize the number of output levels. Other dispositions will be studied in the future. In addition to that only sinusoidal modulation was applied for this study case for simplicity, while other modulating strategies with common-mode injections, typical for classical MMC, are possible as well.

As it was pointed out in Section II, each interleaved submodule can produce additional $K+1$ voltage levels. Therefore, in total, the implemented hybrid modulation scheme (cf. Fig. 3) can synthesize $2 K N+1$ levels [8]. The arm modulation signals, generated by the control scheme described in Section III.A, are compared with corresponding arm carrier signals. For instance, the upper arm ISM-MMC modulation signal is compared with the upper arm carrier signals $\left(h_{11}, \ldots h_{N K}\right)$. The output of the comparator block is a set $\mathbb{D}_{u x}$ of logical PWM signals $\left(d_{11}, \ldots d_{N K}\right)$. Similarly, the lower arm PWM set $\mathbb{D}_{l x}$ is generated. Later, these two sets are applied to the voltage balancing strategy as discussed in Section III.A.

Another characteristic that should be discussed for the implemented hybrid modulation scheme is the switching frequency of ISM-MMC. It is well-known that depending on whether arm interleaving is applied or not the switching frequency of classical MMC can be defined either $2 N f_{s w \mid S M}$ or $N f_{s w \mid S M}$, respectively. The term $f_{s w \mid S M}$ represents the switching frequency of a submodule. For PS-PWM it is equal to the carrier frequency $\left(f_{c}\right)$, while for LS-PWM it can be calculated as $f_{c} / N$. In this context, the hybrid modulation scheme (PD LS-PWM + PS-PWM) will result in the converter's switching frequency $2 K f_{c}$.

\section{Simulation RESUlts}

In this section, numerical simulation results are presented to demonstrate the operation behavior of the proposed ISM-MMC. The comparison of working characteristics between standard MMC and newly introduced topology are made basing on the converter structures given in Fig. 1. As a base architecture, an ISMMMC with 2 SMs per arm $(N=2)$ and 3 interleaved HB-legs in each SM $(K=3)$ was selected. Depending on which carrier frequency was applied 1 or $0.333 \mathrm{kHz}$, it is labeled as "N2K3f1k" or "N2K3f333", respectively. A classical MMC, having 2 SMs per $\operatorname{arm}(N=2)$ with 3 parallel switches, which commutate simultaneously at $f_{c}=1 \mathrm{kHz}$, is labeled as "N2Kp3f1k". Another MMC with 6 SMs per arm $(N=6)$ and $f_{c}=1 \mathrm{kHz}$ is labeled as "N6K1f1k". These labels are used throughout the whole paper for short notation of the compared configurations. These configurations have been chosen to demonstrate main differences between the classical MMC and ISM-MMC having similar design parameters (i.e., individual SM capacitance, total number of switches, etc.) or output characteristics (i.e., number of ac voltage levels, etc.). The system example was selected in relation to a real design of the front-end converter in ultra-fast electric vehicle (EV) chargers (i.e., "Terra 184" ABB Ltd.). The main system parameters of compared configurations are listed in Table I. It should be noted that ISM-MMC is easily scalable to any voltage and current levels, therefore, other EV charger designs with few MW power and more can be realized (i.e., "NBSK1000" Power Electronics Corp., "1.5MW Charger" Proterra Corp.). Proper selection of ISM-MMC design parameters (i.e., number of series connected SMs, number of interleaved HB-legs in each SM, etc.) is an optimization problem that includes many variables, for example, cost and power capability of the power electronic switches. This topic is beyond the scope of this article and will be reported in detail in future works.

Performance of the compared configurations was firstly verified

TABLE I. MAIN SYSTEM PARAMETERS FOR COMPARED CONFIGURATIONS

\begin{tabular}{|c|c|c|c|c|c|}
\hline Description & Symbol & N2K3f1k & N2K3f333 & N2Kp3f1k & N6K1f1k \\
\hline number of SM in each arm & $N$ & 2 & 2 & 2 & 6 \\
\hline number of HB-legs in each SM & $K$ & 3 & 3 & 1 (3 paral. sw.) & 1 \\
\hline dc output power and dc-link voltage & $P_{d c}, V_{d c}$ & \multicolumn{4}{|c|}{$180 \mathrm{~kW}, 1000 \mathrm{~V}$} \\
\hline rated ac input power, current (rms) & $S_{a c}, i_{x}$ & \multicolumn{4}{|c|}{$214 \mathrm{kVA}, 310 \mathrm{~A}$} \\
\hline ac line-to-line voltage (rms) and fundamental frequency & $v_{x y}, f$ & \multicolumn{4}{|c|}{$400 \mathrm{~V}, 50 \mathrm{~Hz}$} \\
\hline sorting frequency & $f_{\text {sort }}$ & \multicolumn{4}{|c|}{$333 \mathrm{~Hz}$} \\
\hline carrier frequency & $f_{c}$ & $1 \mathrm{kHz}$ & $333 \mathrm{~Hz}$ & $1 \mathrm{kHz}$ & $1 \mathrm{kHz}$ \\
\hline $\begin{array}{l}\text { equivalent arm inductor / } \\
\text { individual interleaved inductor (if applicable) }\end{array}$ & $\begin{array}{c}R_{\text {arm }}, L_{\text {arm }} / \\
\quad R, L \\
\end{array}$ & $\begin{array}{r}4 \mathrm{~m} \Omega \\
6 \mathrm{~m} \Omega, 2.5 \mathrm{mH} \\
\end{array}$ & $103.3 \mathrm{~A} \mathrm{rms})$ & $4 \mathrm{~m} \Omega, 1$ & $\begin{array}{c}\mathrm{mH}(@ 310 \mathrm{Arms}) / \\
-\end{array}$ \\
\hline equivalent arm capacitance / individual SM capacitance & $C_{\text {arm }} / C, E S R$ & \multicolumn{3}{|c|}{$3.2 \mathrm{mF} / 6.4 \mathrm{mF}, 0.2 \mathrm{~m} \Omega$} & $3.2 \mathrm{mF} / 19.2 \mathrm{mF}, 0.2 \mathrm{~m} \Omega$ \\
\hline IGBT module (Infineon Technologies AG) & - & \multicolumn{3}{|c|}{ FF150R12RT4 } & FF450R07ME4 \\
\hline
\end{tabular}


under open-loop control operation mainly to demonstrate differences of ac voltage characteristics (cf. Fig. 4) and to introduce behavioral relation between sorting frequency, which is used in the capacitor voltage balancing algorithm, and proper current sharing among interleaved HB-legs in each SM (cf. Fig. 5). It must be noted that although this section refers to "open-loop control" operation, nevertheless, the internal control methods (cf. Fig. 3), such as arm-energy control, circulating current control and capacitor voltage sorting algorithm, are enabled.

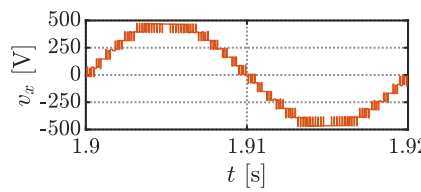

(a)

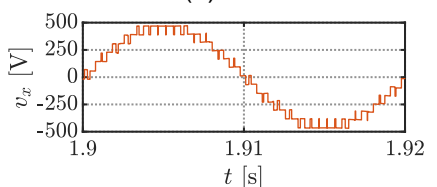

(c)

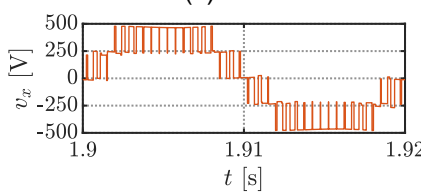

(e)

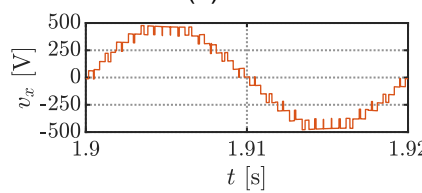

(g)

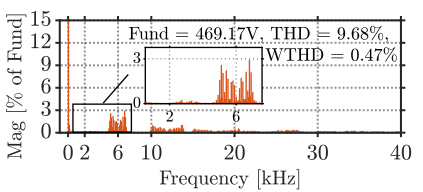

(b)

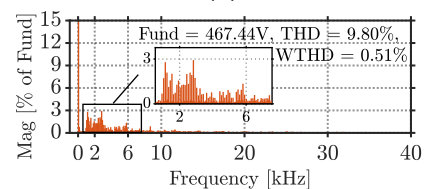

(d)

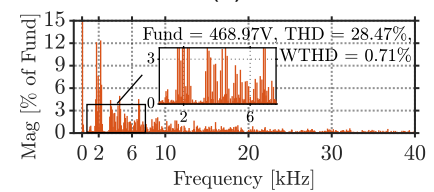

(f)

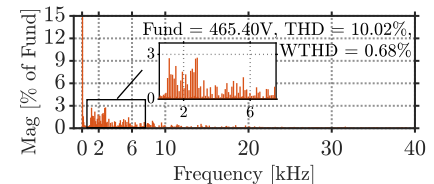

(h)
Fig. 4. Phase voltage and its corresponding harmonic content in ISMMMC (a-d) and classical MMC (e-h) for the following subcases: $(a, b)$ N2K3f1k; (c,d) N2K3f333; (e,f) N2Kp3f1k; (g,h) N6K1f1k.

For this test, the compared topologies were working in inverter mode delivering power from dc to ac side. The reference output power was selected similar to the system design example (cf. Table I), namely $180 \mathrm{~kW}$ operating with unity power factor. To depict the maximum of available ac phase voltage levels, the highest modulation index from linear modulation range of the sinusoidal PWM (without overmodulation) was selected. To speed up convergence of output characteristics to steady state values after the start-up, a higher value of internal resistance of interleaved inductors was set $\left(R_{\text {arm }}=156.4 \mathrm{~m} \Omega / R=234.7 \mathrm{~m} \Omega\right.$ where it is applicable). Considering internal resistances of the other components (i.e., IGBT modules, capacitors) they remain unchanged. The increase results in a higher equivalent arm resistance and consequently larger voltage drop. This effect is well noticeable in Fig. 4. However, it does not introduce tremendous effect on the performed comparative analysis. As expected, configurations "N2K3f1k", "N2K3f333" and "N6K1f1k" can generate 13 voltage levels operating under PD LS-PWM scheme. It is interesting to notice here that "N2K3f333" and "N6K1f1k" have quite similar harmonic spectrum with dominant switching harmonic components appearing as a first sideband at $2 \mathrm{kHz}$. This effect was explained in Section III.B. On the other hand, "N2K3f1k" with similar THD exhibits superior performance since the first sideband harmonics are located around $6 \mathrm{kHz}$, consequently reducing requirements for the ac interface filter. In this context, weighted THD (WTHD) [24] can quantitatively justify greater performance of "N2K3f1k" in comparison with other converter arrangements. At the same time "N2Kp3f1k" having the same number of SMs (capacitors) and power switches that work without interleaving scheme can synthesize only 5 voltage levels with remarkably high harmonic pollution.

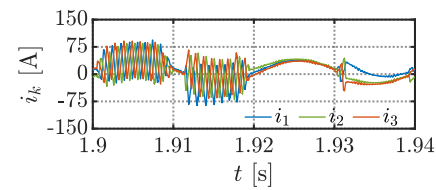

(a)

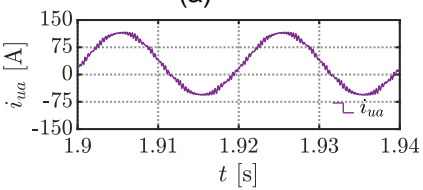

(b)

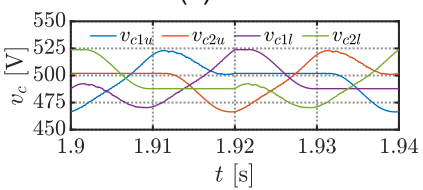

(c)

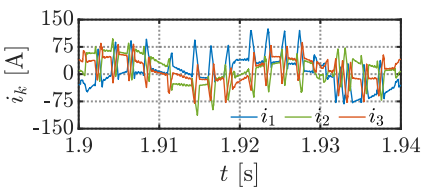

(d)

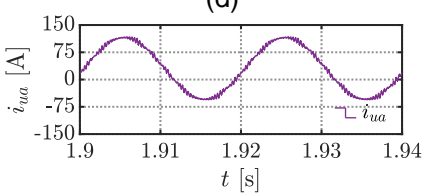

(e)

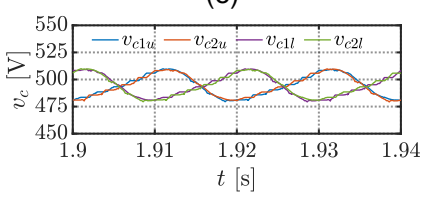

(f)
Fig. 5. Interleaved leg currents in one submodule of ISM-MMC $(a, d)$, the corresponding arm current (b,e) and capacitor voltages in phase ' $a$ ' of ISM-MMC (c,f) at sorting frequency $50 \mathrm{~Hz}(\mathrm{a}, \mathrm{b}, \mathrm{c})$ and at $1 \mathrm{kHz}(\mathrm{d}, \mathrm{e}, \mathrm{f})$.

Another noteworthy characteristic of ISM-MMC is the relation between sorting frequency of capacitor voltage balancing algorithm, and equal current sharing among interleaved HB-legs in each SM. It is well visible from Fig. 5 that operating with low sorting frequency of voltage balancing function, better current distribution among interleaved legs can be achieved. Conversely, higher sorting frequency results in higher imbalance of the currents in interleaved legs. This fact can be explained by significant time constants of interleaved inductors and large number of commutations within one fundamental period provoked by capacitor voltage balancing algorithm. This aspect must be taken into account while selecting sorting frequency. It is also evident that the output SM's current (i.e., the arm current) is not affected by the high magnitude current ripple that present in the interleaved currents. It is canceled out due to summation of the interleaved currents. Similarly, it does not appear in the output phase current, as well as the circulating current that is part of the arm current (cf. dc offset in Fig. 5b,e).

\section{HARDWARE-IN-THE-LOOP TESTS}

The HIL tests are designed to demonstrate dynamic behavior of the newly proposed ISM-MMC and verify applicability of the classical MMC control techniques for the novel topology. Therefore, the HIL simulation results are presented for "N2K3f1k" case only. The HIL tests have been performed using RT Box2 (Plexim) in the PLECS environment with sampling period $12.5 \mu$ s. Fig. 6 presents a view of the HIL setup and circuit scheme of the tested converter. To reduce the computation burden of the HIL setup, for these tests a single-phase ISM-MMC with 2 SMs per arm $(N=2)$ and 3 interleaved legs in each SM $(K=3)$ was used. The real-time simulation was performed in a multitasking mode, distributing tasks between available CPUs, 
such that power circuit was emulated on CPU1 and CPU2, while the entire control has been deployed on CPU3.

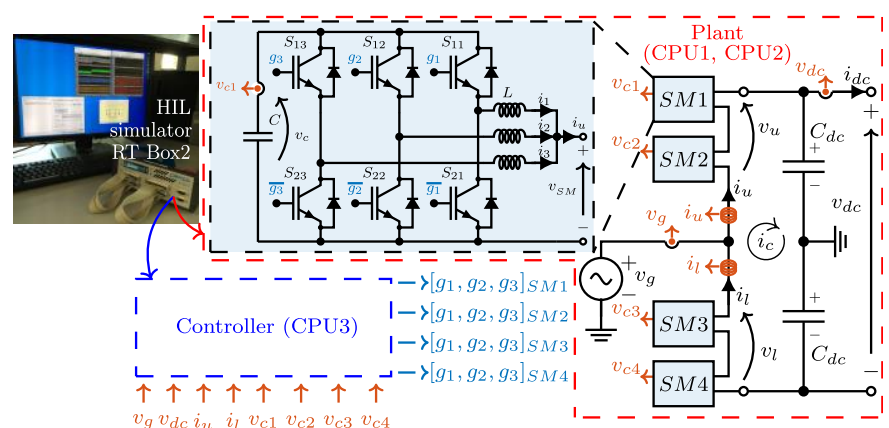

Fig. 6. View of the HIL simulator and circuit scheme of the single-phase ISM-MMC.

The single-phase, grid-connected (230 Vrms) ISM-MMC supplies a dc load $(60 \mathrm{~kW})$ with unity power factor via the dclink. To provide a reference neutral wire connection, a split dclink capacitor $\left(C_{d c}=15 \mathrm{mF}\right)$ has been used. The dc load was realized as a controlled current source with known demand profile. The profile includes a step-like change of dc current by $50 \%$ from half to full demand and back. The second dc current step is applied when the system has already experienced a steplike drop of dc-link voltage by $10 \%$. The internal resistance of interleaved inductors was set to a higher value $\left(R_{\text {arm }}=30.3 \mathrm{~m} \Omega /\right.$ $R=45.5 \mathrm{~m} \Omega$ ) to smooth divergence of interleaved currents from one another since the interleaved currents are not actively controlled. This resistance value includes also on-state resistance of an IGBT. The rest parameters of passive components $(L, C$, cf. Table I) were assigned with reference to real commercial components having standard tolerances. For $R, L$ the tolerance is $15 \%$, while for $C$ it is $10 \%$. Accounting of these tolerances in simulations was made by generating random values following a Gaussian distribution and having a confidence interval of $\pm 4 \sigma$.

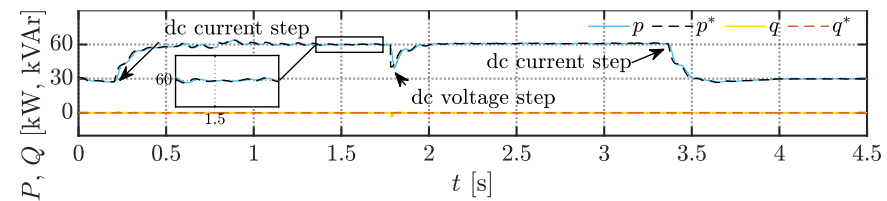

i

(a)

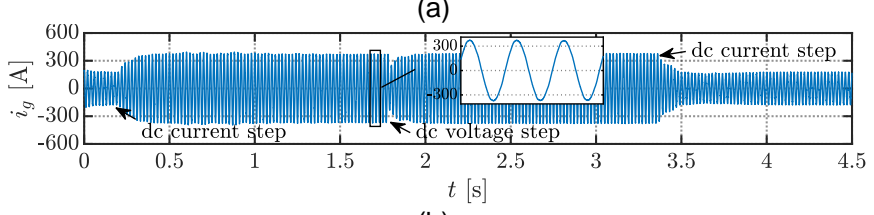

(b)

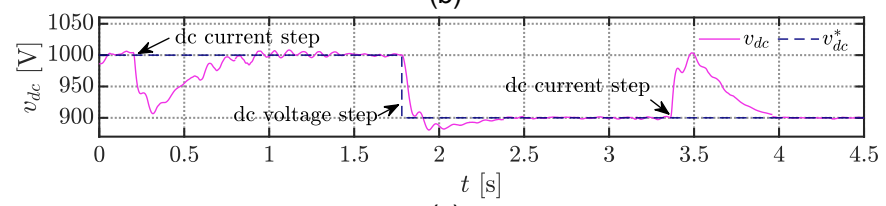

(c)

Fig. 7. Active and reactive powers (a) supplied by the grid (solid traces) along with their reference values (dashed lines), ac phase current (b) and dc-link voltage (c) - measured value (solid trace) and its reference (dashed trace).

Fig. 7 depicts measured ac powers (active and reactive) supplied by the grid, the corresponding ac phase current, and dclink voltage along with its reference. Fig. 7a confirms that the HIL simulated ISM-MMC operates with unity power factor (supplied reactive power is zero).

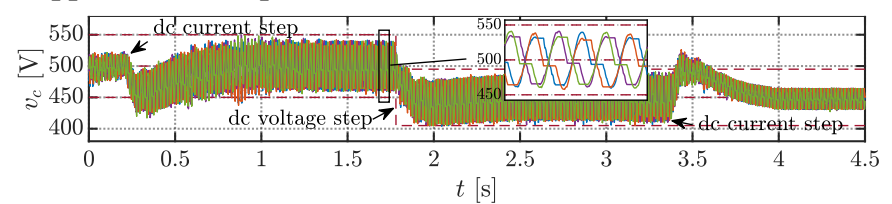

(a)

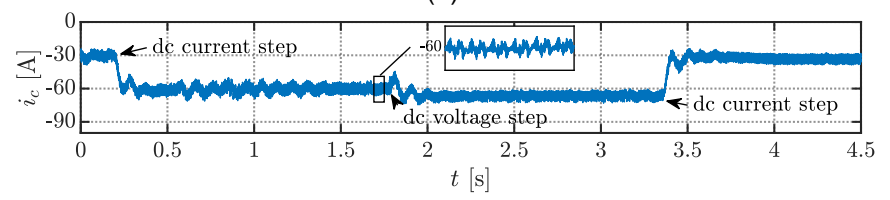

(b)

Fig. 8. Capacitor voltages (a) from each submodule of the ISM-MMC (solid traces) along with its $\pm 10 \%$ tolerance band and mean value (dashed lines) and corresponding circulating current (b) in phase leg of ISM-MMC.

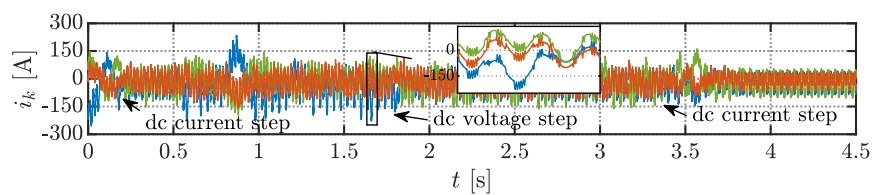

(a)

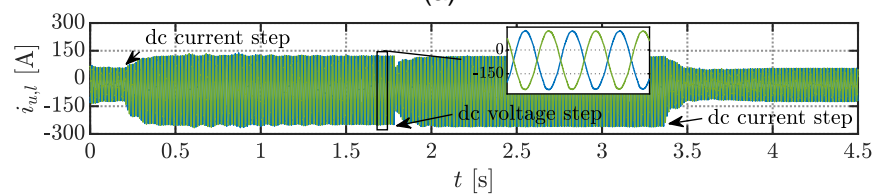

(b)

Fig. 9. Currents in interleaved legs of the submodule SM1 (a) and arm currents of ISM-MMC (b).

Fig. 8a presents measured capacitor voltages from each submodule of the single-phase ISM-MMC. The dashed lines in this plot represent $\pm 10 \%$ voltage ripple tolerance band and mean value of capacitor voltage. Dynamic behavior of circulating current in response of the imposed system changes can be seen from Fig. 8b. Circulating current after some transients reaches steady state values depending on the operating point. The circulating current is composed of dc current and high frequency ripple component. Overall, the system behavior under classical MMC close-loop control demonstrates expected, stable performance in all tested operational modes having unequal parameters of passive components.

Fig. 9 confirms the fact that the balancing of interleaved currents is a decoupled control task and should be implemented individually in each SM. Balancing of interleaved currents as it was mentioned previously is outside the scope of this paper and will be discussed in detail in future works. Nevertheless, it worth to notice that even though the currents inside of a SM are unbalanced and feature high-magnitude ripple, the sum of them (arm current) remains balanced and sinusoidal-like.

\section{COMPARISON}

This section gives a comparative analysis of major features of the converter configurations listed in Table I. The first aspect to be compared is the number of main components (i.e., IGBT modules, inductors, capacitors, etc.) and their characteristics (current and voltage ratings, etc.). All configurations have the same number of power switches, while arrangement and operation modes are different. The two chosen reference IGBT modules are from the 
same generation, device family and manufactured by the same company. The "FF150R12RT4" module is designed with the following maximum rated values: collector-emitter voltage $1200 \mathrm{~V}$ and continuous dc current 150A. Similarly, the "FF450R07ME4" has the following maximum ratings: $650 \mathrm{~V}$ and $450 \mathrm{~A}$, respectively. Configurations "N2K3f1k" and "N2K3f333" feature distributed inductor arrangement, having 6 inductors in total per arm. In contrast, "N2Kp3f1k" and "N6K1f1k" have only one inductor per arm. Although structural characteristics of these inductors are different (internal resistance and inductances), one should note that distributed (interleaved) inductors carry only a portion of arm current, thus, can be designed with a significantly smaller crosssection of composed wires. This fact directly reflects on cost, weight and volume of the converter. To form either ISM-MMC ("N2K3f1k" and "N2K3f333") or classical MMC ("N2Kp3f1k") configurations with 2 SMs per arm only 2 capacitors per arm are needed. In fact, for high current applications those SM capacitors are composed of a set of parallel connected capacitors. However, for simplicity it will be assumed that the SM capacitors are single components. For the "N6K1f1k" configuration 6 capacitors per arm are required. Yet, to keep voltage ripple across the capacitors within $\pm 10 \%$ tolerance band of its average value, the size of capacitors must be increased drastically. As a matter of fact, the classical MMC configuration is not well suitable for low-voltage, high-power applications, since an increase of ac voltage levels results in a corresponding increment of series connected SMs in each arm, while low dc-link voltage significantly reduces allowed fluctuating voltage range of SM capacitors, therefore, a bigger capacitor is required in each SM.

Analytical developments for the converter efficiency have been discussed in literature in great detail (e.g. [25]). Therefore, for sake of conciseness, the efficiency formulation is omitted in this paper. Instead, the focus has been given to the comparison between studied configurations. Nevertheless, some assumptions must be taken into consideration in the analytical derivations of the converter efficiency, namely constant capacitor voltage in each SM and almost sinusoidal (ripple magnitude is negligible) balanced currents in the interleaved HB-legs. The main parameters used for efficiency analysis are listed in Table I.

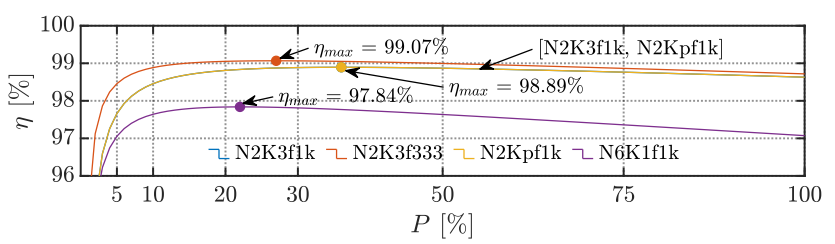

Fig. 10. Converter efficiency for compared cases.

Fig. 10 depicts efficiencies for the compared converter configurations. Firstly, arrangements "N2K3f1k" and "N2Kp3f1k" have identical efficiency curve since they have similar circuital structure and operating switching frequencies, leading to indistinguishable conduction and switching losses. The only difference among them is the interleaving effect, which does not affect efficiency but only improves the output characteristics as demonstrated in the previous section. Instead, "N2K3f333" operates with three times reduced switching frequency, inducing fewer switching losses in comparison to "N2K3f1k" or "N2Kp3f1k". A significantly lower efficiency can be observed in the "N6K1f1k" case due to higher conductions losses which is the consequence of the large number of series submodules.

Overall, it is evident from the analysis that the ISM-MMC can offer higher or equal efficiency in comparison with classical MMC, while having the same or enhanced ac characteristics of the converter, depending on operating switching frequency.

\section{CONCLUSION}

A new interleaved submodule structure has been proposed for modular multilevel converters, forming a novel topology named ISM-MMC. Among strong points of the new converter structure are easily scalable voltage and current ratings, suitable for all voltage levels high power applications, enhanced output waveforms, improved efficiency and fault tolerance capability. The latter concept justified by highly modular structure of SMs in ISM-MMC exploiting benefits of parallel systems (term in readability studies), where failure of a single component (HBleg) does not mean failure of whole system (SM). On the contrary some drawbacks are increased complexity of the converter architecture with many commutating devices to be controlled, proper current sharing between interleaved legs within SM, larger number of required inductors. Nevertheless, it should be pointed out that a distributed arrangement of these inductors is not necessarily a weak point since the current ratings of the inductors is proportionally less than a classical arm inductor. Therefore, with an optimal design equal or superior qualitive characteristics (cost, weight, volume, etc.) can be reached.

A proper modulation scheme has been presented consisting of level shifted PWM for controlling the submodules and phase shifted PWM for driving the different legs inside each submodule. In addition to that the applicability of classical MMC control techniques for ISM-MMC has been proven.

Numerical simulations and HIL tests were carried out to validate key features of ISM-MMC and the implemented modulation technique, eventually proving feasibility of the proposed MMC-based structure with reference to ultrafast EV charging infrastructure. Then, an efficiency comparison between several classical MMC and ISM-MMC configurations is presented by showing a relative efficiency gain up to $1.56 \%$ (with total power losses reduction up to $53.24 \%$ ) depending on the compared configurations and operating power level.

A future development could refer to an optimal design of ISMMMC, considering cost and characteristics of the composing components (i.e., less SMs/interleaved legs with higher ratings or more SMs/interleaved legs with reduced ratings). In addition, a proper design of interleaved inductors must be studied, considering maximum allowed peak-to-peak current ripple in interleaved assemblies and output characteristics of the converter.

\section{REFERENCES}

[1] L. Zhang et al., "Modeling, control, and protection of modular multilevel converter-based multi-terminal HVDC systems: A review," CSEE J. Power Energy Syst., vol. 3, no. 4, pp. 340-352, Dec. 2017.

[2] F. Briz, M. Lopez, A. Rodriguez, and M. Arias, "Modular power electronic transformers: Modular multilevel converter versus cascaded h-bridge solutions," IEEE Ind. Electron. Mag., vol. 10, no. 4, pp. 6-18, Dec. 2016.

[3] M. A. Perez, S. Ceballos, G. Konstantinou, J. Pou, and R. P. Aguilera, "Modular Multilevel Converters: Recent Achievements and Challenges," IEEE Open J. Ind. Electron. Soc., vol. 2, pp. 224-239, Feb. 2021.

[4] S. Milovanovic and D. Dujic, "On Power Scalability of Modular Multilevel 
Converters: Increasing Current Ratings through Branch Paralleling," IEEE Power Electron. Mag., vol. 7, no. 2, pp. 53-63, Jun. 2020.

[5] J. W. Kolar and G. Ortiz, "Solid-State-Transformers: Key Components of Future Traction and Smart Grid Systems," in Proc. Power Electron. Conf., 2014, pp. 22-35.

[6] K. Drobnic et al., "An Output Ripple-Free Fast Charger for Electric Vehicles Based on Grid-Tied Modular Three-Phase Interleaved Converters," IEEE Trans. Ind. Appl., vol. 55, no. 6, pp. 6102-6114, Nov. 2019.

[7] F. M. Alhuwaishel, A. K. Allehyani, S. A. S. Al-Obaidi, and P. N. Enjeti, "A Medium-Voltage DC-Collection Grid for Large-Scale PV Power Plants with Interleaved Modular Multilevel Converter," IEEE J. Emerg. Sel. Top. Power Electron., vol. 8, no. 4, pp. 3434-3443, Dec. 2020.

[8] A. Viatkin, M. Ricco, R. Mandrioli, T. Kerekes, R. Teodorescu, and G. Grandi, "Modular Multilevel Converters Based on Interleaved Half-Bridge Submodules," in Proc. IEEE Int. Conf. Ind. Technol. 2021, pp. 440-445.

[9] K. Sharifabadi, L. Harnefors, R. Teodorescu, H.-P. Nee, and S. Norrga, Design, Control, and Application of Modular Multilevel Converters for HVDC Transmission Systems. Wiley-IEEE Press, 2016.

[10] Sixing Du, Apparao Dekka, Bin Wu, and Navid Zargari, Modular Multilevel Converters: Analysis, Control, and Applications. Wiley-IEEE Press, 2018.

[11] M. Ricco, L. Mathe, E. Monmasson, and R. Teodorescu, "FPGA-Based Implementation of MMC Control Based on Sorting Networks," Energies, vol. 11, no. 9, p. 2394, Sep. 2018.

[12] M. Ricco, L. Mathe, M. Hammami, F. Lo Franco, C. Rossi, and R. Teodorescu, "A Capacitor Voltage Balancing Approach Based on Mapping Strategy for MMC Applications," Electronics, vol. 8, no. 4, p. 449, Apr. 2019.

[13] B. Li, R. Yang, D. Xu, G. Wang, W. Wang, and D. Xu, "Analysis of the phase-shifted carrier modulation for modular multilevel converters," IEEE Trans. Power Electron., vol. 30, no. 1, pp. 297-310, 2015.

[14] P. Sochor and H. Akagi, "Theoretical and Experimental Comparison between Phase-Shifted PWM and Level-Shifted PWM in a Modular Multilevel SDBC Inverter for Utility-Scale Photovoltaic Applications," IEEE Trans. Ind. Appl., vol. 53, no. 5, pp. 4695-4707, Sep. 2017.

[15] P. Hu and D. Jiang, "A level-increased nearest level modulation method for modular multilevel converters," IEEE Trans. Power Electron., vol. 30, no. 4, pp. 1836-1842, Apr. 2015.

[16] G. Konstantinou, J. Pou, S. Ceballos, R. Darus, and V. G. Agelidis, "Switching Frequency Analysis of Staircase-Modulated Modular Multilevel Converters and Equivalent PWM Techniques," IEEE Trans. Power Deliv., vol. 31, no. 1, pp. 28-36, Feb. 2016.

[17] Y. Deng and R. G. Harley, "Space-vector versus nearest-level pulse width modulation for multilevel converters," IEEE Trans. Power Electron., vol. 30, no. 6, pp. 2962-2974, Jun. 2015.

[18] Y. Deng, Y. Wang, K. H. Teo, and R. G. Harley, "A simplified space vector modulation scheme for multilevel converters," IEEE Trans. Power Electron., vol. 31, no. 3, pp. 1873-1886, Mar. 2016.

[19] A. Dekka, B. Wu, N. R. Zargari, and R. L. Fuentes, "A Space-Vector PWM-Based Voltage-Balancing Approach with Reduced Current Sensors for Modular Multilevel Converter," IEEE Trans. Ind. Electron., vol. 63, no. 5, pp. 2734-2745, May 2016.

[20] G. J. Capella, J. Pou, S. Ceballos, G. Konstantinou, J. Zaragoza, and V. G. Agelidis, "Enhanced phase-shifted PWM carrier disposition for interleaved voltage-source inverters," IEEE Trans. Power Electron., vol. 30, no. 3, pp. 1121-1125, Mar. 2015.

[21] D. Zhang, F. Wang, R. Burgos, and D. Boroyevich, "Common-mode circulating current control of paralleled interleaved three-phase two-level voltage-source converters with discontinuous space-vector modulation," IEEE Trans. Power Electron., vol. 26, no. 12, pp. 3925-3935, 2011.

[22] B. Cougo, G. Gateau, T. Meynard, M. Bobrowska-Rafal, and M. Cousineau, "PD modulation scheme for three-phase parallel multilevel inverters," IEEE Trans. Ind. Electron., vol. 59, no. 2, pp. 690-700, Feb. 2012.

[23] G. Konstantinou, G. J. Capella, J. Pou, and S. Ceballos, "Single-Carrier Phase-Disposition PWM Techniques for Multiple Interleaved VoltageSource Converter Legs," IEEE Trans. Ind. Electron., vol. 65, no. 6, pp. 4466-4474, Jun. 2018.

[24] D. G. Holmes and T. A. Lipo, Pulse Width Modulation for Power Converters Principles and Practice. Wiley-IEEE Press, 2010.

[25] L. Bieber, J. Pfannschmidt, L. Wang, A. Nami, and W. Li, "A Hybrid Three-Level and Modular Multilevel Converter With DC Fault Blocking Capability and Reduced Semiconductor Losses," IEEE Trans. Power Deliv., vol. 35, no. 4, pp. 1895-1908, Aug. 2020. 